\title{
A Practical Technique for Gait Recognition on Curved and Straight Trajectories
}

\author{
Fatimah Abdulsattar ${ }^{1,2}$ \\ ${ }^{1}$ Southampton University \\ Southampton, UK \\ fsalg12@ecs.soton.ac.uk
}

\author{
John Carter ${ }^{1}$ \\ ${ }^{2}$ Al-Mustansiriya University \\ Baghdad , Iraq \\ jncdecs.soton.ac.uk
}

\begin{abstract}
Many studies show the effectiveness of gait in surveillance and access control scenarios. However, appearance changes due to walking direction changes impose a challenge for gait recognition techniques that assume people only walk in a straight line. In this paper, the effect of walking along straight and curved path is studied by proposing a practical technique which is based on the three key frames in the start, middle and end of the gait cycle. The position of these frames is estimated in 3D space which is then used to estimate the local walking direction in the first and second part of the cycle. The technique used $3 D$ volume sequences of the people to adapt to changes in the walking direction. The performance is evaluated using a newly collected dataset and the Kyushu University 4D Gait Dataset, containing people walking in straight lines and curves. With the proposed technique, we obtain a correct classification rate of $98 \%$ for matching straight with straight walking and $81 \%$ for matching straight with curved walking averaged over both datasets. The variation in walking patterns when a person walks along a straight or curved path is most likely to be responsible for the difference. In support of this, the recognition rate when matching curved with curved walking is $99 \%$ on our dataset.
\end{abstract}

\section{Introduction}

Human identification techniques have important applications in surveillance and access control scenarios. Gait is an attractive biometric that can be used in these scenarios as it does not require direct interaction with or cooperation from the subjects and can be recorded at a distance.

One of the challenges for gait-based human identification techniques is appearance change due to walking direction changes with respect to the camera [see Fig. 1], also known as view variation. Several techniques have been proposed to tackle this issue, but the majority of them study walking in a straight line. This is the simplest style of walking. However, it rarely happens in daily life since walking paths tend to be curves rather than straight lines [12], but most work has assumed the latter.

One class of view invariant recognition learns the mapping/relationship of gait sequences from different views. In [6], the authors modeled the relationship between gaits from different views based on multiple groups of the motion coclustering using Canonical Correlation Analysis. In [10] a View Transform Model (VTM) was used to transform gait features from different views into the same view. The performance of these techniques was degraded when used to recognise untrained views. Recently, Muramatsu et al. [11] proposed an arbitrary VTM to recognise untrained view. These do not consider curved trajectories.

This work and others build gait models from a set of calibrated cameras. In [4], a recursive image synthesised technique based on estimating foot points from each frame in one gait cycle was proposed. 3D volumetric data reconstructed from multi-camera setup were used for training and a single camera for testing. Although this technique was applied to curved trajectories by fitting a 2D curve to the estimated foot points, it appears to be computationally expensive and has not been tested from an arbitrary viewpoint. The technique in [8] proposed Gait Entropy Volume by exploiting the concept of entropy on reconstructing volumes from a set of synchronised cameras. This technique achieved promising results for gait recognition in unconstrained paths. López-Fernández, D. et al. [9] introduced rotation invariant gait descriptor to tackle the problem of rotation changes on curved trajectories. The main limitation of the techniques in $[8,9]$ is that they require a multi-camera setup for training and testing.

In this paper, we presented a new practical technique of gait recognition which allows identifying people regardless of walking direction changes and is independent of the camera's viewing angle. This method uses $3 \mathrm{D}$ volumes of the people in the gallery and an alignment procedure which requires only the position of the three key frames at the start, middle and end of a gait cycle in a 3D space. Accordingly, the local walking direction is estimated in the first and second part of a gait cycle. Each subject image is compared 


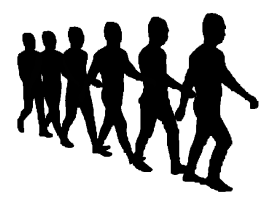

(a) Straight walk

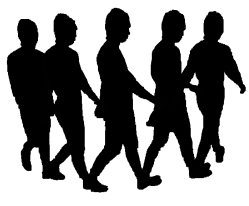

(b) Curved walk
Figure 1. Appearance changes due to walking direction changes [taken from [4]]

with the corresponding synthetic image generated from 3D volumes. The method is validated using a newly collected multi-view gait dataset of 50 people and the Kyushu University 4D Gait Dataset. Several analyses have been conducted to demonstrate the effectiveness of this technique.

\section{Proposed technique}

The method starts with the detection of three key frames at the start, middle and end of a gait cycle, dividing the gait cycle into two parts. We assume that the motion in the first and second part of a gait cycle can be approximated as linear even if the walking path is a curve. First, the position of a person in these frames is determined in 3D space. Then, the local walking direction in each part of a gait cycle is calculated. This method uses $3 \mathrm{D}$ volumetric data to synthesise reference silhouettes independent of changes in the walking directions. The details of the proposed method will be explained in the following subsections.

\subsection{Key frames selection}

During walking, the body of the subject will swing to the left or right depending on which foot is moving forward even if he/she walks in a straight line. The swing will reach a maximum when the two feet are at full extension (double support stance). On the other hand, when the two feet completely overlap (mid stance) the swing towards left or right will be at the minimum. Three such consecutive frames will be chosen as the key frames to mark the start, middle and end of a gait cycle.

\subsection{Estimating positions and walking direction}

In this section, we will describe how to extract the position and walking direction (a) from the 2D subject silhouettes and (b) from the 3D volumes of people.

\section{(a) from the 2D subject silhouettes}

We assume that the subject walks on a flat floor with a known location in 3D space and that the camera is calibrated. The $3 \mathrm{D}$ position of the subject in a $2 \mathrm{D}$ image is estimated from the projection of the location of his/her feet onto the floor. As the position should be estimated for the subject in the three key frames in a gait cycle where the two feet completely overlap, the global minimum $C\left[x, y_{\text {min }}\right]$ of the boundary silhouette points should be located. How- ever estimating the $3 \mathrm{D}$ foot position from only a single point, as does in [4], may not be reliable due to imperfections in silhouette extraction. Therefore, most of the points $\left\{C[x, y], y_{\min } \leq y \leq y_{\min }+\Delta y\right\}$ in the foot region are determined as shown in Fig. 2. We set $\Delta y$ to 5. These points are then projected onto the floor to calculate their positions in $3 \mathrm{D}$ space using a camera projection matrix. Finally, the point,$f$, that minimises the total distance to all the projected foot points is chosen as the $3 \mathrm{D}$ foot position

$$
\min _{f} \sum\left\|f_{j}-f\right\|, \quad j=1,2 \ldots J
$$

where $\|$.$\| is the Euclidean distance and J$ is the number of the projected foot points $f_{j}$.

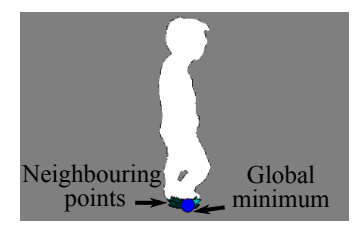

Figure 2. Foot points detection

As the motion in the first and second part of a gait cycle is assumed to be linear, the subject's position in the intermediate frames is determined by fitting two line segments in the two parts of a gait cycle using linear interpolation as

$$
\begin{array}{cc}
f_{1 i}=f_{s}+i \times \frac{f_{m}-f_{s}}{L_{1}-1} & i=0,1 \ldots L_{1}-1 \\
f_{2 i}=f_{m}+i \times \frac{f_{e}-f_{m}}{L_{2}-1} & i=0,1 \ldots L_{2}-1
\end{array}
$$

where $f_{s}, f_{m}$ and $f_{e}$ are the $3 \mathrm{D}$ foot positions at the start, middle and last frame in a gait cycle. $L_{1}$ and $L_{2}$ are the number of frames in the first and second part of a cycle. $f_{1 i}$ and $f_{2 i}$ are the 3D foot position at each frame in the first and second part of a cycle respectively. The local walking directions (viewing angles) in the two parts of a gait cycle are then calculated from the gradient of the fitted lines.

\section{(b) from the 3D volumes of people}

To calculate the pose of each $3 \mathrm{D}$ volume along one gait cycle, the foot position of the three key volumes at the start, middle and end of the cycle is first computed and the local walking direction in the first and second part of the cycle is then estimated. To determine the foot position of each key volume, the lower $(11 \%)$ part of the $3 \mathrm{D}$ volume is mapped onto the floor using an orthogonal top projection to extract the foot region. This step aims to remove the effect of the upper limb movement. After that, the point $(F)$ that minimised the sum of Euclidean distances to all other points in the foot region is calculated as a foot position of the volume. Next, the foot positions of the intermediate volumes in the 
two parts of a gait cycle are computed by fitting two line segments using linear interpolation as

$$
\begin{array}{cc}
F_{1 i}=F_{s}+i \times \frac{F_{m}-F_{s}}{N 1-1} & i=0,1 \ldots N_{1}-1 \\
F_{2 i}=F_{m}+i \times \frac{F_{e}-F_{m}}{N 2-1} & i=0,1 \ldots N_{2}-1
\end{array}
$$

where $F_{1 i}$ and $F_{2 i}$ are the foot position of the volumes in the first and second part of a 3D gait cycle respectively, $F_{s}$, $F_{m}$ and $F_{e}$ are the foot position of the first, middle and last volume in a gait cycle. $N_{1}$ and $N_{2}$ are the number of the volumes in the first and second part of a gait cycle. Next, the gradient of the two fitted lines are used to calculate the local walking directions (viewing angles) in the first and second part.

\subsection{D alignment and synthetic image generation}

In order to align the position and orientation of each $3 \mathrm{D}$ volume in one gait cycle with that of the corresponding subject image, we normalise the number of frames in all gait cycles such that the phases in both $3 \mathrm{D}$ and $2 \mathrm{D}$ gait cycles are matched. Here, we normalise the number of frames in the first and second parts of a 3D gait cycle using $i_{1}^{\prime}=i_{1} \times N_{1} / N_{1}$ and $i_{2}=i_{2} \times N_{2} / N_{2}$ where $N_{1}$ and $N_{2}$ are the required number of frames in the first and second parts of the 3D cycle respectively. The number of frames in a 2D gait cycle is normalised in a similar manner. To align the orientation, a 3D coordinate system is set up at each foot position and then a $3 \mathrm{D}$ volume is rotated around the vertical axis (Z-axis). The volumes in the first part of a gait cycle are rotated by $\left(\varphi_{1}-\delta_{1}\right)$ and in the second part by $\left(\varphi_{2}-\delta_{2}\right)$ where $\varphi_{1}$ and $\delta_{1}$ are the viewing angles in the first part of a $2 \mathrm{D}$ and $3 \mathrm{D}$ gait cycle respectively while $\varphi_{2}$ and $\delta_{2}$ are the viewing angles in the second part of a $2 \mathrm{D}$ and $3 \mathrm{D}$ gait cycle respectively. To align the position, each volume in the first part of a gait cycle is translated by $T_{1_{i}}$ and in the second part by $T_{2_{i}}$ as

$$
T_{1_{i}}=f_{1_{i}}-F_{1_{i}} \quad, \quad T_{2_{i}}=f_{2_{i}}-F_{2_{i}}
$$

Finally, each aligned volume is projected onto a 2D image plane using the camera projection matrix to produce the synthetic image. This is the most computationally intense part of the algorithm, but it could be carried out effectively by commonly available graphics cards if required [5].

\subsection{Gait features extraction}

The pose of the subject and synthetic silhouettes varies from frame to frame. Therefore, the gait features will be extracted on a frame-by-frame basis. First, the silhouette image is cropped by placing a bounding box of a fixed size such that the centroid of a silhouette is in the middle of a cropped image. No height normalisation is performed as the height can be an important discriminative feature and this process can distort the shape of silhouettes when the orientation changes. Then, the cropped silhouette is divided into several areas and the features are derived from each area to provide a detailed description and concatenated to form the final feature vector. Fig. 3 shows the divisions that are considered in our experiments.

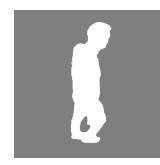

(a) 1 area

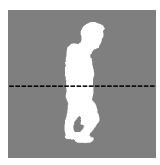

(b) 2 areas

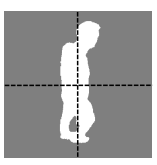

(c) 4 areas

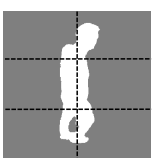

(d) 6 areas

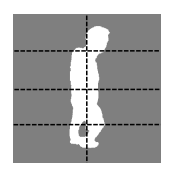

(e) 8 areas

Figure 3. The divided areas in a silhouette

We used Generic Fourier Descriptors (GFDs) as our gait features since GFDs are invariant to many geometric distortions (e.g. translation, scale and rotation) including a small amount of perspective, and have some intrinsic resistance to noise [13]. These invariant properties can be useful to tackle a gap that may result in foot estimation from $2 \mathrm{D}$ and $3 \mathrm{D}$ data. In these descriptors, multi-resolution fine features in both radial and circular directions can be captured and only a small number of features is required to efficiently describe the shape. The first step to derive the GFDs is to convert the image $a[x, y]$ from Cartesian coordinates to polar coordinates $A[r, \theta]$ where $[x, y] \equiv[r \sin \theta, r \cos \theta], r$ and $\theta$ are the polar coordinates and $x$ and $y$ are the Cartesian coordinates of the image. The origin of the polar space is set to the centroid of the silhouette to account for translation invariance. The Polar Fourier Transform $(P)$ is applied as

$P(\rho, \phi)=\sum_{r=0}^{R-1} \sum_{i=0}^{K-1} A\left(r, \theta_{i}\right) \times \exp \left[-j 2 \pi\left(\frac{r}{R} \rho+\frac{2 \pi i}{K} \phi\right)\right]$

where $\theta_{i}=i(2 \pi / K), 0 \leq \rho \leq R, 0 \leq \phi \leq K . R$ and $K$ are the radial and angular resolutions respectively. The rotation invariance is obtained by using the magnitude of the coefficients. Finally, the GFDs are computed by normalising the magnitude of the first coefficient by the polar image area and the magnitudes of the remaining coefficients by the magnitude of the first coefficient to account for scale invariance. We calculated only the first 60 GFDs (4 radial and 15 angular frequencies) from each area in the silhouette.

\subsection{Gait classification}

Frame-by-frame comparison can be affected by errors in gait cycle estimation. To reduce the effect of these errors, we propose the following procedure to compute the distance between two gait cycles. The feature vector of each frame is compared against the features of its corresponding frame, the frame before and the frame after. We then take the minimum, 
$D_{i}$

$$
D_{i}=\min \left(\left\|p_{i}-g_{i+j}\right\|\right), j \in-1,0,1
$$

where $p$ and $g$ are the feature vector of probe and gallery respectively and $i$ and $i+j$ refer to specific frames. At the ends of the cycle, only 2 comparisons will be made. Then, the total distance between two cycles of length $Z$ is computed as

$$
\operatorname{Dist}(p, g)=\sum_{i=0}^{Z-1} D_{i}
$$

\section{Gait datasets}

Two multi-view gait datasets that represented different situations were used to evaluate the performance of our method. The first dataset was recorded in a narrow corridor to simulate the narrow walkway in airports (access control). In this dataset, there are 12 synchronised cameras distributed on two walls as well as 2 unsynchronised (arbitrary) wide-angle cameras as shown in Fig. 4. The first arbitrary camera is placed close to the middle of the walking path at a height of $2.38 \mathrm{~m}$ to capture the subject's image from the side while the second arbitrary camera is positioned near the end of the walking path at a height of $1.75 \mathrm{~m}$ to capture the subject's image from the rear. The sequences are captured by all cameras at a rate of 30 frames/seconds. The resolution of video sequences captured by the synchronised cameras and the side-view camera is $640 \times 480$ pixels while the resolution for the rear-view camera is $1024 \times 768$ pixels. Fifty subjects participated in the data capturing process where each subject walked 6 times along a straight line and 6 times on a curved trajectory in the middle of the corridor. The $3 \mathrm{D}$ volumes of people are reconstructed from all synchronised cameras using a visual hull strategy [7] to build a gallery while the image from the unsynchronised cameras are used as a probe.

The second dataset is the Kyushu University 4D Gait Database (KY4D) [4]. This dataset consists of video sequences for 42 people recorded by 16 synchronised cameras, which are placed around a wide circular studio of radius $3.5 \mathrm{~m}$ as shown in Fig. 5. The sequences were captured at a rate of 20 frames/second and had a resolution of $1032 \times 776$ pixels. Each subject walked 4 times along a straight path and once on each of 2 circles of radius $3 \mathrm{~m}$ and $1.5 \mathrm{~m}$. Although this dataset includes $3 \mathrm{D}$ volumes reconstructed using the images from all the cameras, we have reconstructed them by excluding the probe (target) camera.

\section{Results}

In the following subsections, we analysed the performance of our technique for two types of walk (straight and curved) using 2 gait datasets: our dataset and KY4D dataset. We used K-Nearest Neighbour classification (KNN) with leave-one-out recognition strategy. All the results in the experiments were evaluated at $K=1$. For each test sequence

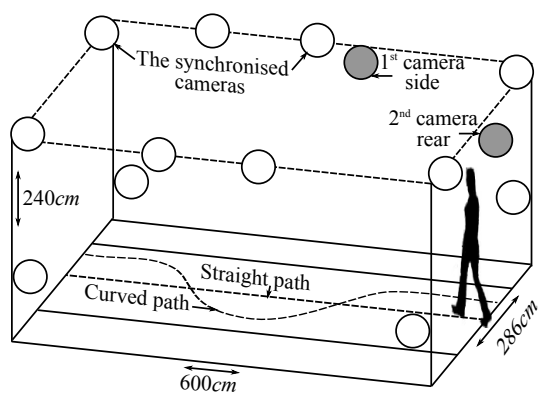

Figure 4. Camera positions and trajectories in our experiment

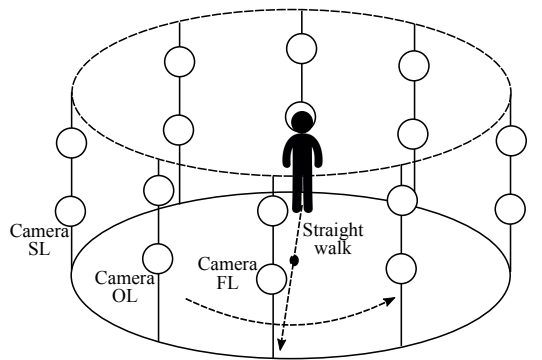

Figure 5. Circular studio for KY4D dataset

during the recognition, we removed the corresponding 3D sequence that was captured at the same time.

\subsection{Analysis of our dataset}

We investigated 2 scenarios for gallery and probe in our dataset: straight-straight (i.e. straight walk data in the gallery and straight walk data in the probe) and straight-curved (i.e. straight walk data in the gallery and curved walk data in the probe). In each scenario, the performance was measured with respect to the number of divided areas in a silhouette and the number of frames per gait cycle.

Firstly, to measure the recognition rate with respect to the number of divided areas in a silhouette, we set the number of frames in a cycle to 30 because this number matches the average gait cycle length measured at 30 frames/second. Fig. 6 shows the Correct Classification Rate (CCR) for straight-straight and straight-curved. The performance for the straight-straight scenario is generally high for all number of divisions and the best result was obtained at 4 divisions. However, the performance for the straight-curved scenario is lower than for the straight-straight scenario but the enhanced result is still observed at 4 divisions.

Secondly, we measured the performance when the number of divisions is 4 and the number of frames in a gait cycle is varied from 5 to 50 but we noticed that there is no improvement in performance beyond 30 frames. This is probably because our sampling procedure adds little additional information when the desired length is greater than the actual length. Therefore, in Table 1 we displayed the recognition results for the straight-straight and straight-curved scenarios 


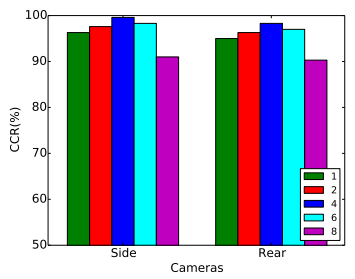

(a) Straight-straight

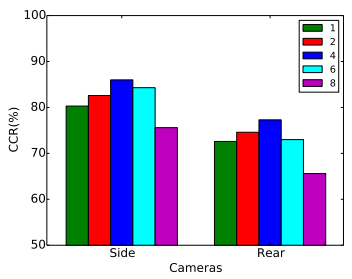

(b) Straight-curved
Figure 6. CCR versus number of divided areas.

versus number of frames up to 30 . From this Table, we can see that the performance was not significantly influenced by the number of frames in a gait cycle. The results show the same trend as in Fig. 6. For comparison purposes, we evaluated the performance using Gait Energy Image (GEI) [3] because it is one of the conventional methods that exhibits high recognition capability and is commonly used for comparison. The averaged recognition rate from the two cameras is $81.4 \%$ for the straight-straight scenario and is $45.6 \%$ for the straight-curved scenario. The performance of GEI is significantly inferior to that of our technique.

Table 1. CCR(\%) versus number of frames/cycle.

\begin{tabular}{c|c|c|c|c|c|c}
\hline & \multicolumn{7}{c}{ Straight-straight } \\
\hline \multirow{2}{*}{ Camera } & \multicolumn{7}{c}{ Number of frames } \\
\cline { 2 - 7 } & $\mathbf{5}$ & $\mathbf{1 0}$ & $\mathbf{1 5}$ & $\mathbf{2 0}$ & $\mathbf{2 5}$ & $\mathbf{3 0}$ \\
\hline Side & 98.3 & 99.0 & 99.3 & 99.3 & 99.6 & 99.6 \\
Rear & 97.3 & 98.0 & 98.0 & 98.3 & 98.3 & 98.3 \\
\hline \multicolumn{7}{c}{ Straight-curved } \\
\hline \multirow{2}{*}{ Camera } & $\mathbf{5}$ & $\mathbf{1 0}$ & $\mathbf{1 5}$ & $\mathbf{2 0}$ & $\mathbf{2 5}$ & $\mathbf{3 0}$ \\
\cline { 2 - 7 } & $\mathbf{5}$ & 84.0 & 85.0 & 85.0 & 86.0 \\
Side & 78.6 & 84.0 & 84.0 & 77.3 \\
\hline
\end{tabular}

To show which process mainly contributes to recognition performance improvement, we evaluated the performance using GFDs without and with the 3D alignment. The GFDs were calculated by dividing each silhouette into 4 divisions and extracting the first 60 coefficients from each division. The averaged recognition rate increased from $71.8 \%$ to $98.9 \%$ for the straight-straight scenario and from $37.5 \%$ to $81.6 \%$ for the straight-curved scenario. These results reflect the huge improvement in performance due to the $3 \mathrm{D}$ alignment since the significant variations in the appearance of silhouettes in the gallery and probe were removed by this process. The built-in-invariance of the features can also compensate for small appearance variations due to errors in foot estimation.

\subsection{Analysis of KY4D dataset}

We applied our technique to the KY4D gait dataset to compare its performance with the most related technique in the literature [4] and with the GEI. We recorded the performance in 2 scenarios as explained in section 4.1. In each scenario, we set the number of divisions in each silhouette to 6 and the number of frames per a gait cycle to 20 which gave the optimum results during the experimental stage.

In the straight-straight scenario, the images of the subject were captured from each of the 3 cameras which showed approximately front (FL), oblique (OL) and side views (SL), as shown in Fig. 5. Table 2 summarises the recognition results for straight-straight scenario from different techniques. The performance of our method is comparable to that in [4] and is better than GEI. However, our technique depends only on the three key frames and does not require recursive implementation as does in [4]. We noticed that the performance of GEI is lower in our dataset than that in KY4D dataset for the straight-straight scenario. This is probably because our dataset has more extreme variation in silhouette shapes in one gait cycle due to the close distance to the cameras and the nature of the wide-angle lens.

Table 2. CCR for straight-straight in KY4D dataset.

\begin{tabular}{l|c|c|c}
\hline Methods & FL & OL & SL \\
\hline ours & 97.6 & 99.4 & 98.2 \\
method [4] & 99.4 & 96.4 & 98.2 \\
GEI & 96.4 & 95.2 & 97.0 \\
\hline
\end{tabular}

For the straight-curved scenario, the probe images were captured by camera FL for people walking along 2 circles of radius (3) and (1.5). Table 3 shows the recognition results where the performance is generally lower than that of the straight-straight scenario. Our method outperforms that of [4] and GEI for people walking on the big circle. We noticed that GEI has the worst performance for both curved trajectories. This is perhaps because the spatio-temporal pattern derived by GEI is negatively affected by the walking direction changes from frame to frame as the corresponding body parts cannot be matched.

Table 3. CCR for straight-curved in KY4D dataset.

\begin{tabular}{c|c|c|c}
\hline Radius & method [4] & GEI & ours \\
\hline $\mathbf{3}$ & 71.4 & 33.3 & 76.1 \\
$\mathbf{1 . 5}$ & 61.9 & 23.8 & 54.8 \\
\hline
\end{tabular}

\subsection{Curved against curved walk matching}

The analysis using our dataset and KY4D dataset in section 4.1 and 4.2 showed that recognition results for straightstraight is much better than straight-curved. The authors of [4] claimed that the stride length of people walking along a curved trajectory is smaller than when walking a long straight line. However, other differences between walking along a straight line and walking on a curve have been noted in several studies $[12,2,1]$. These differences are (1) the 
body leans towards the center of the curvature, see Fig. 7, (2) the feet move asymmetrically where the outer foot twist more than the inner foot and (3) the head rotates into the direction of the future turn before the trunk. We also noticed these in the data and these imply that directly comparing straight walks with curved walks is not appropriate. This suggests that using curved walks in the gallery may lead to improve the performance with curved probes. Therefore, we measured the performance when the curved walk data was used in both gallery and probe. For our dataset, we set the number of frames to 30 and the number of silhouette divisions to 4 . The CCR for the side and rear cameras were $100 \%$ and $98.6 \%$ respectively. The performance on KY4D was not recorded by [4] for this scenario. To evaluate the performance for KY4D dataset, we used 20 frames per gait cycle and 6 divisions in a silhouette. Since there is only one sequence for each of the two circular paths, we used the sequences for the big circle to recognise the small circle sequences and used the sequences for the small circle to identify the big circle sequences. The CCR from the front view camera FL for the first and second cases were $76.1 \%$ and $80.9 \%$ respectively. These results are considerably higher than those for straight-curved scenario in both datasets.

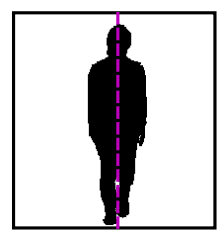

(a) Straight walk

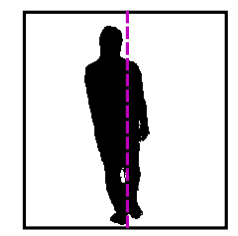

(b) Curved walk
Figure 7. How the body pose differs during a curved walk, when viewed from straight ahead.

\section{Conclusions}

In this paper, a new practical technique has been proposed to recognise people regardless of their walking direction or viewpoint of the camera. The technique depends on estimating the positions of the three key frames in one gait cycle which have shown to be sufficient to do the $3 \mathrm{D}$ alignment to tackle the problem of appearance changes. The results show that the recognition rate of our technique obtains up to $98 \%$ and $90 \%$ for people walking along straight and curved trajectories respectively when the same walking trajectory is used in both gallery and probe. However, the recognition rate decreases to $80 \%$ when different walking trajectories are used for enrolment and recognition. We believed that the discrepancy in the gait patterns of the subject walking across different trajectories is most likely to blame as shown in Fig. 7. The results also confirm this as the recognition rate of our technique obtains $99 \%$ on our dataset when using curved walk in both the gallery and probe.
The performance of our technique compares favourably with [4]. However, our technique does not require a recursive implementation to precisely align each frame in one gait cycle.

Acknowledgements We would like to thank the Higher Committee of Education Development in Iraq for funding this study.

\section{References}

[1] G. Courtine. Tuning of a basic coordination pattern constructs straight-ahead and curved walking in humans. Journal of Neurophysiology, 91(4):1524-1535, 2004.

[2] G. Courtine and M. Schieppati. Human walking along a curved path. ii. gait features and emg patterns. European Journal of Neuroscience, 18(1):191-205, 2003.

[3] J. Han and B. Bhanu. Individual recognition using gait energy image. IEEE Transactions on Pattern Analysis and Machine Intelligence, 28(2):316-322, 2006.

[4] Y. Iwashita, K. Ogawara, and R. Kurazume. Identification of people walking along curved trajectories. Pattern Recognition Letters, 48:60-69, 2014.

[5] J. Kruger and R. Westermann. Acceleration techniques for gpu-based volume rendering. In Proceedings of the 14th IEEE Visualization (VIS'03), Washington, DC, USA, 2003.

[6] W. Kusakunniran, Q. Wu, J. Zhang, H. Li, and L. Wang. Recognizing gaits across views through correlated motion co-clustering. IEEE Transactions on Image Processing, 23(2):696-709, 2014.

[7] A. Laurentini. The visual hull concept for silhouette-based image understanding. IEEE Transactions on Pattern Analysis and Machine Intelligence, 16(2):150-162, 1994.

[8] D. López-Fernández, F. Madrid-Cuevas, A. Carmona-Poyato, R. Muñoz-Salinas, and R. Medina-Carnicer. Entropy volumes for viewpoint-independent gait recognition. Machine Vision and Applications, 26(7-8):1079-1094, 2015.

[9] D. López-Fernández, F. Madrid-Cuevas, A. Carmona-Poyato, R. Muñoz-Salinas, and R. Medina-Carnicer. Multi-view gait recognition on curved trajectories. In Proceedings of the 9th International Conference on Distributed Smart Camera, pages 116-121. ACM, 2015.

[10] Y. Makihara, R. Sagawa, Y. Mukaigawa, T. Echigo, and Y. Yagi. Gait recognition using a view transformation model in the frequency domain. In Computer Vision-ECCV, pages 151-163. Springer, 2006.

[11] D. Muramatsu, A. Shiraishi, Y. Makihara, M. Uddin, and Y. Yagi. Gait-based person recognition using arbitrary view transformation model. IEEE Transactions on Image Processing, 24(1):140-154, 2015.

[12] M. Sreenivasa, I. Frissen, J. Souman, and M. Ernst. Walking along curved paths of different angles: the relationship between head and trunk turning. Experimental Brain Research, 191(3):313-320, 2008.

[13] D. Zhang and G. Lu. Shape-based image retrieval using generic fourier descriptor. Signal Processing: Image Communication, 17(10):825 - 848, 2002. 